\title{
Geometrical Nonlinearity and Stability Analyses of Stiffened and Unstiffened Laminated Composite Plates Using High Order Element
}

\author{
M.M. Hegaze
}

\begin{abstract}
Stiffened plates are used in several applications, such as aircrafts, ships and aerospace structures. Geometric nonlinearity and stability analyses of stiffened and unstiffened composite laminated plate are presented in this paper using the high order finite element. Strain energy method has been used in the derivation. The strain energy has been divided into infinitesimal strain energy for small deformation and finite strain energy fro large deformation. The shear effect has been taken into consideration in the element derivation. The coupling effect of small and large deformation on the strain energy has been introduced to define the finite force vector. The results of the proposed element derivation have been validated through the comparison with published, software (ANSYS-12) and experimental results with geometric nonlinearity and stability analyses. Parametric investigations have been carried out on different case studies with different boundary conditions, number of layers, stacking sequence and aspect ratio.
\end{abstract}

Keywords: Composite material; Finite element method; geometric nonlinearity, stability analysis

\section{Introduction}

The technology of composite materials has experienced a rapid development. The main reason for this development is requirements for high performance materials, especially in military applications and aerospace structures. Plates are the most commonly structural forms used in these applications. Linear analysis of plates using finite element method was formulated by Zienkiewicz [1] using different types of elements.

The study of the review articles and other recent publications reveals that geometrical nonlinear problem for laminated composite plates even now have attracted the attention of many researchers and investigators. In particular cases, geometrical nonlinearity is discussed to introduce the effect of large deformation based on several approaches and using different elements (Mindlin, Serendipity ... etc.) [2-3].

In the literature, basically two different theories were used in order to study laminated composite plates; the classical laminated plate theory and the shear deformation plate theories. The effect of transverse shear stress is neglected in the classical theory which can be used for thin plates. The transverse shear strains in composite layered plates are very effective parameters to estimate an accurate deflection and stresses. The first order shear deformation theory was proposed by Mindlin [4] and Reissner [5] where the shear effect was taken into consideration and the normal to the midsurface remain line but not normal.

\footnotetext{
*Egyptian Armed Forces, Egypt
} 
Ferreira and Barbosa [6] used finite element model to present a geometric non-linear analysis of composite shell structures. The material was assumed to have an orthotropic behavior and the performance of the model has been tested in some buckling examples. In the same field of buckling analysis, Istvan and Laszlo [7] introduced formulas for the calculation of the buckling load of rectangular orthotropic plates with clamped and/or simply supported edges.

The higher-order shear deformation theory was proposed to satisfy the plate boundary conditions and to explain the plate deformation which can be used for different types of analyses (frequency analysis, buckling analysis ... etc.) [8-10].

An analytical approach for buckling analysis of thick functionally graded rectangular plates is presented by Bodaghia and Saidi [8] where the equilibrium and stability equations are derived according to the higher-order shear deformation plate theory.

The onset of buckling in square laminated multi-layered composite plates, subject to unidirectional in-plane loads, is investigated by Fiedler, et. [9] within the framework of a generalized higher-order shear deformation theory suitable to capture significant transverse shear and thickness-wise deformation effects. The displacement field is expanded in a Taylor series of the thickness coordinate with arbitrary polynomial degree.

From the previous review it can be conclude that the higher-order theory for the analysis of laminated plates has received increased attention in the last decade. The aim of this paper is to provide a geometric nonlinear and stability analyses of composite laminated plate using a new derivation. The proposed element derivation based on the strain energy method which can be divided into infinitesimal and finite strain energy which corresponding to infinitesimal stiffness and stress matrices. This technique gives us the ability to avoid the problem of unsymmetry of stiffness matrix due to large deformation effect. A new derivation of high order shear element has been discussed. The effects of number of layers and degree of orthotropy are studied with different case studies by different boundary conditions and aspect ratio. The accuracy of the proposed derivation of the element has been verified through the comparison of the results with published, software (ANSYS-12) and experimental results.

\section{Nodal Displacement Formulation}

Plates are structural elements which are usually defined in terms of a midplane and thickness distribution. It may be classified into thin or thick according to the ratio of the thickness to span length. The displacement components at a general point may be resolved into in-plane displacements $\mathrm{u}, \mathrm{v}$ and out-of plane displacement $\mathrm{w}$ which can be expressed as;

$$
\left.\begin{array}{l}
u=\mathrm{u}_{0}+\mathrm{z} \theta_{\mathrm{y}}+\mathrm{f}(\mathrm{z}) \psi_{\mathrm{y}} \\
v=\mathrm{v}_{0}-\mathrm{z} \theta_{\mathrm{x}}-\mathrm{f}(\mathrm{z}) \psi_{\mathrm{x}} \\
w=\mathrm{w}_{0}
\end{array}\right\}
$$

where $\mathrm{u}_{0}, \mathrm{v}_{0}, \mathrm{w}_{0}$ are the displacement components of the midplane in $\mathrm{x}, \mathrm{y}$, and $\mathrm{z}$ directions and $\psi_{x}$ and $\psi_{y}$ are the additional rotations due to transverse shear.

For an n-node element, the degrees of freedom at any point on the midplane are defined in terms of in-plane displacements, out-of plane displacements, and transverse shear strain. The nodal displacement vector of the element (at time t) can be defined as follows: 


$$
\underline{\delta}(\mathrm{t})=\left\{\underline{\delta}_{0}(\mathrm{t}) \quad \underline{\delta}_{\mathrm{b}}(\mathrm{t}) \quad \underline{\delta}_{t}(\mathrm{t})\right\}
$$

where $\underline{\delta}_{0}(\mathrm{t})=\left\{\left(u_{0}\right) \quad\left(v_{0}\right)\right\}_{\text {for } \mathrm{n} \text { node element }}$

$$
\begin{aligned}
& \underline{\delta}_{\mathrm{b}}(\mathrm{t})=\left\{\begin{array}{llll}
\left(w_{0}\right) & \left(\theta_{x 0}\right) & \left(\theta_{y 0}\right) & \left(\theta_{z 0}\right)
\end{array}\right\}_{\text {fornnodeelement }} \\
& \underline{\delta}_{t}(\mathrm{t})=\left\{\left(\psi_{x}\right)\left(\psi_{y}\right)\right\}_{\text {forn nodeelement }}
\end{aligned}
$$

\section{Strain-Displacement Relations}

For simplification of derivations, the strains are defined in terms of two separate vectors:

(i) x-y components vector $\underline{\varepsilon}=\left\{\begin{array}{lll}\varepsilon_{x} & \varepsilon_{y} & \gamma_{\mathrm{xy}}\end{array}\right\}^{T}$

(ii) Transverse shear vector $\gamma=\left\{\begin{array}{ll}\gamma_{x z} & \gamma_{y z}\end{array}\right\}^{T}$

Then according to Green's strain displacement equations the vector of $\mathrm{x}-\mathrm{y}$ strain components can be expressed in terms of displacement components as follows [11]:

$$
\left.\begin{array}{l}
\varepsilon_{x}=\frac{\partial \mathrm{u}}{\partial \mathrm{x}}+\frac{1}{2}\left[\left(\frac{\partial \mathrm{u}}{\partial \mathrm{x}}\right)^{2}+\left(\frac{\partial \mathrm{v}}{\partial \mathrm{x}}\right)^{2}+\left(\frac{\partial \mathrm{w}}{\partial \mathrm{x}}\right)^{2}\right] \\
\varepsilon_{y}=\frac{\partial \mathrm{v}}{\partial \mathrm{y}}+\frac{1}{2}\left[\left(\frac{\partial \mathrm{u}}{\partial \mathrm{y}}\right)^{2}+\left(\frac{\partial \mathrm{v}}{\partial \mathrm{y}}\right)^{2}+\left(\frac{\partial \mathrm{w}}{\partial \mathrm{y}}\right)^{2}\right] \\
\gamma_{x y}=\frac{\partial \mathrm{u}}{\partial \mathrm{y}}+\frac{\partial \mathrm{v}}{\partial \mathrm{x}}+\left[\left(\frac{\partial \mathrm{u}}{\partial \mathrm{x}}\right)\left(\frac{\partial \mathrm{u}}{\partial \mathrm{y}}\right)+\left(\frac{\partial \mathrm{v}}{\partial \mathrm{x}}\right)\left(\frac{\partial \mathrm{v}}{\partial \mathrm{y}}\right)+\left(\frac{\partial \mathrm{w}}{\partial \mathrm{x}}\right)\left(\frac{\partial \mathrm{w}}{\partial \mathrm{y}}\right)\right]
\end{array}\right\}
$$

Hence, the strain-displacement equations can be rewritten in matrix form as follows;

$$
\underline{\varepsilon}=\underline{\varepsilon}_{\mathrm{S}}+\underline{\varepsilon}_{\mathrm{L}}
$$

where the subscript "s" represents small strains and "L" represents large strains.

$$
\begin{aligned}
& \underline{\varepsilon}_{\mathrm{S}}=\underline{\varepsilon}_{0}-\mathrm{z} \underline{\varepsilon}_{\mathrm{b}}+\mathrm{g}(\mathrm{z}) \underline{\varepsilon}_{\mathrm{t}} \\
& \underline{\varepsilon}_{\mathrm{L}}=\underline{\varepsilon}_{\mathrm{m}}+\underline{\varepsilon}_{\mathrm{w}}+\mathrm{z}^{2} \underline{\varepsilon}_{\theta}+\mathrm{g}^{2}(\mathrm{z}) \underline{\varepsilon}_{\psi}-\mathrm{z} \underline{\varepsilon}_{\mathrm{m} \theta}+\mathrm{g}(\mathrm{z}) \underline{\varepsilon}_{\mathrm{m} \psi}+\mathrm{zg}(\mathrm{z}) \underline{\varepsilon}_{\theta \psi}
\end{aligned}
$$

where the infinitesimal strain components can be defined in terms of nodal displacements and strain shape function matrices $\underline{B}$ as follows:

$$
\left.\begin{array}{ll}
\hat{\gamma}=\underline{\mathrm{B}}_{\gamma \gamma}(x, y) \underline{\delta}_{t} & \underline{\varepsilon}_{0}=\underline{\mathrm{B}}_{00}(x, y) \underline{\delta}_{0} \\
\underline{\varepsilon}_{\mathrm{b}}=\underline{\mathrm{B}}_{b b}(x, y) \underline{\delta}_{b}+\underline{\mathrm{B}}_{b t}(x, y) \underline{\delta}_{t} & \underline{\varepsilon}_{\mathrm{t}}=\underline{\mathrm{B}}_{t t}(x, y) \underline{\delta}_{t}
\end{array}\right\}
$$

where 


$$
\begin{aligned}
& \underline{\mathrm{B}}_{y y}(x, y)=\left[\begin{array}{cccc}
0 & -N_{1} & \ldots & \ldots \\
N_{1} & 0 & \ldots & \ldots
\end{array}\right] \quad \underline{\mathrm{B}}_{00}(x, y)=\left[\begin{array}{ccccc}
N_{1, x} & 0 & \ldots & \ldots \\
0 & N_{1, y} & \ldots & \ldots \\
N_{1, y} & N_{1, x} & \ldots & \ldots
\end{array}\right] \quad \underline{\mathrm{B}}_{t t}=\left[\begin{array}{cccc}
0 & N_{1, x} & \ldots & \ldots \\
-N_{1, y} & 0 & \ldots & \ldots \\
-N_{1, x} & N_{1, y} & \ldots & \ldots
\end{array}\right] \\
& \underline{\mathrm{B}}_{b b}(x, y)=\left[\begin{array}{cccccc}
F_{1, x x} & H_{1, x x} & -G_{1, x x} & P_{1, x x} & \ldots & \ldots \\
F_{1, y y} & H_{1, y y} & -G_{1, y y} & P_{1, y y} & \ldots & \ldots \\
2 F_{1, x y} & 2 H_{1, x y} & -2 G_{1, x y} & 2 P_{1, x y} & \ldots & \ldots
\end{array}\right] \underline{\mathrm{B}}_{b t}(x, y)=\left[\begin{array}{cccc}
-H_{1, x x} & G_{1, x x} & \ldots & \ldots \\
-H_{1, y y} & G_{1, y y} & \ldots & \ldots \\
-2 H_{1, x y} & 2 G_{1, x y} & \ldots & \ldots
\end{array}\right]
\end{aligned}
$$

The finite strain components can also be expressed in terms of rotation vectors $\underline{\theta}$ and $\underline{A}$ as follows:

$$
\left.\begin{array}{l}
\underline{\varepsilon}_{\mathrm{m}}=\frac{1}{2} \underline{\mathrm{A}}_{m} \underline{\theta}_{m}=\frac{1}{2} \underline{\mathrm{A}}_{m} \underline{G}_{m} \underline{\delta}_{0} \\
\underline{\varepsilon}_{\theta}=\frac{1}{2} \underline{\mathrm{A}}_{\theta} \underline{\theta}_{\theta}=\frac{1}{2}\left(\underline{\mathrm{A}}_{\theta} \underline{G}_{\theta} \underline{\delta}_{b}+\underline{\mathrm{A}}_{\theta} \underline{G}_{\theta t} \underline{\delta}_{t}\right) \\
\underline{\varepsilon}_{\mathrm{m} \theta}=\underline{\mathrm{A}}_{\theta} \underline{\theta}_{m}=\underline{\mathrm{A}}_{\theta} \underline{G}_{m} \underline{\delta}_{0}=\underline{\mathrm{A}}_{m} \underline{\theta}_{\theta}=\underline{\mathrm{A}}_{m} \underline{G}_{\theta} \underline{\delta}_{b}+\underline{\mathrm{A}}_{m} \underline{G}_{\theta t} \underline{\delta}_{t} \\
\underline{\varepsilon}_{\theta \psi}=-\underline{\mathrm{A}}_{\theta} \underline{\theta}_{\psi}=-\underline{\mathrm{A}}_{\theta} \underline{G}_{\psi} \underline{\delta}_{t}=-\underline{\mathrm{A}}_{\psi} \underline{\theta}_{\theta}=-\underline{\mathrm{A}}_{\psi} \underline{G}_{\theta} \underline{\delta}_{b}-\underline{\mathrm{A}}_{\psi} \underline{G}_{\theta t} \underline{\delta}_{t} \\
\underline{\varepsilon}_{\mathrm{w}}=\frac{1}{2} \underline{\mathrm{A}}_{w} \underline{\theta}_{w}=\frac{1}{2}\left(\underline{\mathrm{A}}_{w} \underline{G}_{w} \underline{\delta}_{b}+\underline{\mathrm{A}}_{w} \underline{G}_{w t} \underline{\delta}_{t}\right) \\
\underline{\varepsilon}_{\psi}=\frac{1}{2} \underline{\mathrm{A}}_{\psi} \underline{\theta}_{\psi}=\frac{1}{2} \underline{\mathrm{A}}_{\psi} \underline{G}_{\psi} \underline{\delta}_{t} \\
\underline{\varepsilon}_{\mathrm{m} \psi}=\underline{\mathrm{A}}_{\psi} \underline{\theta}_{m}=\underline{\mathrm{A}}_{\psi} \underline{G}_{m} \underline{\delta}_{0}=\underline{\mathrm{A}}_{m} \underline{\theta}_{\psi}=\underline{\mathrm{A}}_{m} \underline{G}_{\psi} \underline{\delta}_{t}
\end{array}\right\}
$$

where

$$
\begin{aligned}
& \underline{\theta}_{m}=\left\{\begin{array}{llll}
u_{0, x} & v_{0, x} & u_{0, y} & v_{0, y}
\end{array}\right\} \quad, \quad \underline{\theta}_{\psi}=\left\{\begin{array}{lllll}
\psi_{y, x} & -\psi_{x, x} & \psi_{y, y} & -\psi_{x, y}
\end{array}\right\} \\
& \underline{\theta}_{\theta}=\left\{\begin{array}{llll}
w_{x x} & w_{x y} & w_{x y} & w_{y y}
\end{array}\right\} \quad, \quad \underline{\theta}_{w}=\left\{\begin{array}{ll}
w_{x} & w_{y}
\end{array}\right\} \\
& \underline{A}_{m}=\left[\begin{array}{cccc}
u_{0, x} & v_{0, x} & 0 & 0 \\
0 & 0 & u_{0, y} & v_{0, y} \\
u_{0, y} & v_{0, y} & u_{0, x} & v_{0, x}
\end{array}\right] \quad, \quad \underline{A}_{\theta}=\left[\begin{array}{cccc}
w_{x x} & w_{x y} & 0 & 0 \\
0 & 0 & w_{x y} & w_{y y} \\
w_{x y} & w_{y y} & w_{x x} & w_{x y}
\end{array}\right] \\
& \underline{A}_{\psi}=\left[\begin{array}{cccc}
\psi_{y, x} & -\psi_{x, x} & 0 & 0 \\
0 & 0 & \psi_{y, y} & -\psi_{x, y} \\
\psi_{y, y} & -\psi_{x, y} & \psi_{y, x} & -\psi_{x, x}
\end{array}\right] \quad, \quad \underline{A}_{w}=\left[\begin{array}{cc}
w_{x} & 0 \\
0 & w_{y} \\
w_{y} & w_{x}
\end{array}\right]
\end{aligned}
$$

and the rotation vectors $\underline{\theta}$ can be related to the nodal parameters as;

$$
\begin{array}{ll}
\underline{\theta}_{m}=\underline{G}_{m m} \underline{\delta}_{0} & \underline{\theta}_{w}=\underline{G}_{w w} \underline{\delta}_{b}+\underline{G}_{w t} \underline{\delta}_{t} \\
\underline{\theta}_{\psi}=\underline{G}_{\psi \psi} \underline{\delta}_{t} & \underline{\theta}_{\theta}=\underline{G}_{\theta \theta} \underline{\delta}_{b}+\underline{G}_{\theta t} \underline{\delta}_{t}
\end{array}
$$

where

$$
\underline{\mathrm{G}}_{m m}=\left[\begin{array}{cccc}
N_{1, x} & 0 & \ldots & \ldots \\
0 & N_{1, x} & \ldots & \ldots \\
N_{1, y} & 0 & \ldots & \ldots \\
0 & N_{1, y} & \ldots & \ldots
\end{array}\right] \quad, \quad \underline{\mathrm{G}}_{\psi \psi}=\left[\begin{array}{cccc}
0 & N_{1, x} & \ldots & \ldots \\
-N_{1, x} & 0 & \ldots & \ldots \\
0 & N_{1, y} & \ldots & \ldots \\
-N_{1, y} & 0 & \ldots & \ldots
\end{array}\right]
$$




$$
\begin{aligned}
\underline{\mathrm{G}}_{W w} & =\left[\begin{array}{lllllll}
F_{1, x} & H_{1, x} & -\mathrm{G}_{1, x} & P_{1, x} & \ldots & \ldots \\
F_{1, y} & H_{1, y} & -\mathrm{G}_{1, y} & P_{1, y} & \ldots & \ldots
\end{array}\right], \underline{\mathrm{G}}_{W t}=\left[\begin{array}{llll}
-H_{1, x} & G_{1, x} & \ldots & \ldots \\
-H_{1, y} & G_{1, y} & \ldots & \ldots
\end{array}\right] \\
\underline{G}_{\theta \theta} & =\left[\begin{array}{lllllll}
F_{1, x x} & F_{1, x x} & -G_{1, x x} & P_{1, x x} & \ldots & \ldots \\
F_{1, x y} & F_{1, x y} & -G_{1, x y} & P_{1, x y} & \ldots & \ldots \\
F_{1, x y} & F_{1, x y} & -G_{1, x y} & P_{1, x y} & \ldots & \ldots \\
F_{1, y y} & F_{1, y y} & -G_{1, y y} & P_{1, y y} & \ldots & \ldots
\end{array}\right], \quad \underline{G}_{\theta t}=\left[\begin{array}{llll}
-\mathrm{H}_{1, \mathrm{xx}} & G_{1, x x} & \ldots & \ldots \\
-H_{1, x y} & G_{1, x y} & \ldots & \ldots \\
-H_{1, \mathrm{xy}} & G_{1, x y} & \ldots & \ldots \\
-H_{1, y y} & G_{1, y y} & \ldots & \ldots
\end{array}\right]
\end{aligned}
$$

\section{Strain Energy Equations}

Due to a differential displacement field, the corresponding change of strain energy density is,

$$
\mathrm{d} \overline{\mathrm{U}}=\mathrm{d} \underline{\varepsilon}^{\mathrm{T}} \underline{\sigma}+\mathrm{d} \underline{\gamma}^{\mathrm{T}} \underline{\tau}
$$

where $\underline{\sigma}=\left\{\begin{array}{lll}\sigma_{\mathrm{x}} & \sigma_{\mathrm{y}} & \tau_{\mathrm{xy}}\end{array}\right\}, \quad \underline{\tau}=\left\{\begin{array}{ll}\tau_{\mathrm{xz}} & \tau_{\mathrm{yz}}\end{array}\right\}$ represent $\mathrm{x}-\mathrm{y}$ stress and shear stress vectors respectively.

The stress at any point inside a composite laminate can be related to strain as follows:

$$
\underline{\sigma}=\underline{\mathrm{D}} \underline{\varepsilon} \quad \& \quad \underline{\tau}=\underline{\mu} \underline{\gamma}
$$

where $\underline{\mathrm{D}}, \underline{\mu}$ represent the stiffness matrix of material.

Hence, the $x-y$ stress vector can be partitioned similar to the strain vector in equation (4) as:

$$
\underline{\sigma}=\underline{\sigma}_{S}+\underline{\sigma}_{L}
$$

where $\underline{\sigma}_{S}, \underline{\sigma}_{L}$ represent the stress vectors due to infinitesimal and finite strains, respectively, i.e.

$$
\underline{\sigma}_{\mathrm{S}}=\underline{\mathrm{D}} \underline{\varepsilon}_{\mathrm{S}} \quad \& \quad \underline{\sigma}_{\mathrm{L}}=\underline{\mathrm{D}} \underline{\varepsilon}_{\mathrm{L}}
$$

Hence, the strain energy density can be written as follows:

$$
\mathrm{d} \overline{\mathrm{U}}=\mathrm{d} \underline{\varepsilon}_{\mathrm{S}}^{\mathrm{T}} \underline{\sigma}_{\mathrm{S}}+\mathrm{d} \underline{\varepsilon}_{\mathrm{S}}^{\mathrm{T}} \underline{\sigma}_{\mathrm{L}}+\mathrm{d} \underline{\varepsilon}_{\mathrm{L}}^{\mathrm{T}} \underline{\sigma}+\mathrm{d} \underline{\gamma}^{\mathrm{T}} \underline{\tau}
$$

which can be rewritten as follows:

$$
\mathrm{d} \overline{\mathrm{U}}=\mathrm{d} \overline{\mathrm{U}}_{\mathrm{S}}+\mathrm{d} \overline{\mathrm{U}}_{\mathrm{L}}+\mathrm{d} \overline{\mathrm{U}}_{\mathrm{SL}}
$$

where $d \bar{U}_{S}$ contains infinitesimal strain effect and $d \bar{U}_{L}, d \bar{U}_{S L}$ contain finite strain effect, i.e.

$$
\mathrm{d} \overline{\mathrm{U}}_{\mathrm{S}}=\mathrm{d} \underline{\varepsilon}_{\mathrm{S}}^{\mathrm{T}} \underline{\sigma}_{\mathrm{S}}+\mathrm{d} \underline{\gamma}^{\mathrm{T}} \underline{\tau}, \quad \mathrm{d} \overline{\mathrm{U}}_{\mathrm{L}}=\mathrm{d} \underline{\varepsilon}_{\mathrm{L}}^{\mathrm{T}} \underline{\sigma}, \quad \mathrm{d} \overline{\mathrm{U}}_{\mathrm{SL}}=\mathrm{d} \underline{\varepsilon}_{\mathrm{S}}^{\mathrm{T}} \underline{\sigma}_{\mathrm{L}}
$$

Then, the change of element strain energy is obtained by integrating the strain energy per unit area over the mindplane area as follows: 


$$
\mathrm{dU}=\iint_{\text {Area }}\left[\int_{\mathrm{z}_{\mathrm{L}}}^{\mathrm{z}_{\mathrm{U}}} \mathrm{d} \overline{\mathrm{U}} \mathrm{dz}\right] \mathrm{dxdy}=\iint_{\text {Area }} \mathrm{d} \mathrm{U}^{\prime} \mathrm{dxdy}
$$

\section{Infinitesimal and Stress Stiffness Matrices [10]}

The changes of small and large strain energy density $d \bar{U}_{S}$ and $d \bar{U}_{L}$ can be expressed as follows;

$d \bar{U}_{\mathrm{S}}=\mathrm{d} \underline{\gamma}^{T} \underline{\tau}+\left(\mathrm{d} \underline{\varepsilon}_{o}-\mathrm{z} \mathrm{d} \underline{\varepsilon}_{b}+\mathrm{g}(\mathrm{z}) \mathrm{d} \underline{\varepsilon}_{t}\right)^{T} \underline{\mathrm{D}}\left(\mathrm{d} \underline{\varepsilon}_{o}-\mathrm{z} \mathrm{d} \underline{\varepsilon}_{b}+\mathrm{g}(\mathrm{z}) \mathrm{d} \underline{\varepsilon}_{t}\right)$

Hence,

$$
\begin{aligned}
d U_{S}= & \mathrm{d} \underline{\delta}_{t}^{T} \underline{K}_{r m} \underline{\delta}_{t}+\mathrm{d} \underline{\delta}_{o}^{T} \underline{K}_{\mathrm{oooo}} \underline{\delta}_{o}+\mathrm{d} \underline{\delta}_{b}^{T} \underline{K}_{\mathrm{bbbb}} \underline{\delta}_{b}+\mathrm{d} \underline{\delta}_{b}^{T} \underline{K}_{\mathrm{bbbt}} \underline{\delta}_{t}+ \\
& \mathrm{d} \underline{\delta}_{t}^{T} \underline{K}_{\mathrm{btbb}} \underline{\delta}_{b}+\mathrm{d} \underline{\delta}_{t}^{T} \underline{K}_{\mathrm{btbt}} \underline{\delta}_{t}+\mathrm{d} \underline{\delta}_{t}^{T} \underline{K}_{\mathrm{tttt}} \underline{\delta}_{t}-\mathrm{d} \underline{\delta}_{o}^{T} \underline{K}_{\mathrm{oobb}} \underline{\delta}_{b}- \\
& \mathrm{d} \underline{\delta}_{o}^{T} \underline{K}_{\mathrm{oobt}} \underline{\delta}_{t}-\mathrm{d} \underline{\delta}_{b}^{T} \underline{K}_{\mathrm{bboo}} \underline{\delta}_{o}-\mathrm{d} \underline{\delta}_{t}^{T} \underline{K}_{\mathrm{btoo}} \underline{\delta}_{o}+\mathrm{d} \underline{\delta}_{o}^{T} \underline{K}_{\mathrm{oott}} \underline{\delta}_{t}+ \\
& \mathrm{d} \underline{\delta}_{t}^{T} \underline{K}_{\mathrm{ttoo}} \underline{\delta}_{o}-\mathrm{d} \underline{\delta}_{b}^{T} \underline{K}_{\mathrm{bbtt}} \underline{\delta}_{t}-\mathrm{d} \underline{\delta}_{t}^{T} \underline{K}_{\mathrm{bttt}} \underline{\delta}_{t}-\mathrm{d} \underline{\delta}_{t}^{T} \underline{K}_{\mathrm{tbb}} \underline{\delta}_{b}-\mathrm{d} \underline{\delta}_{t}^{T} \underline{K}_{\mathrm{tbt}} \underline{\delta}_{t}
\end{aligned}
$$

and

$d \bar{U}_{\mathrm{L}}=\left(\mathrm{d} \underline{\varepsilon}_{m}+\mathrm{d} \underline{\varepsilon}_{w}+\mathrm{z}^{2} \mathrm{~d} \underline{\varepsilon}_{\theta}+\mathrm{g}^{2}(\mathrm{z}) \mathrm{d} \underline{\varepsilon}_{\psi}-\mathrm{zd} \underline{\varepsilon}_{m \theta}+\mathrm{g}(\mathrm{z}) \mathrm{d} \underline{\varepsilon}_{m \psi}+\mathrm{zg}(\mathrm{z}) \mathrm{d} \underline{\varepsilon}_{\theta \psi}\right)^{\mathrm{T}} \underline{\sigma}$

Hence,

$$
\begin{aligned}
d U_{L}= & \mathrm{d} \underline{\delta}_{o}^{T} \underline{K}_{\mathrm{mmmm}}^{\sigma} \underline{\delta}_{o}+\mathrm{d} \underline{\delta}_{b}^{T} \underline{K}_{\mathrm{wwww}}^{\sigma} \underline{\delta}_{b}+\mathrm{d} \underline{\delta}_{b}^{T} \underline{K}_{\mathrm{wwwt}}^{\sigma} \underline{\delta}_{t}+\mathrm{d} \underline{\delta}_{t}^{T} \underline{K}_{\mathrm{wtww}}^{\sigma} \underline{\delta}_{b}+ \\
& \mathrm{d} \underline{\delta}_{t}^{T} \underline{K}_{\mathrm{wwwt}}^{\sigma} \underline{\delta}_{t}+\mathrm{d} \underline{\delta}_{b}^{T} \underline{K}_{\theta \theta \theta \theta}^{\sigma} \underline{\delta}_{b}+\mathrm{d} \underline{\delta}_{b}^{T} \underline{K}_{\theta \theta \theta}^{\sigma} \underline{\delta}_{t}+\mathrm{d} \underline{\delta}_{t}^{T} \underline{K}_{\theta \theta \theta}^{\sigma} \underline{\delta}_{b}+ \\
& \mathrm{d} \underline{\delta}_{t}^{T} \underline{K}_{\theta \theta t}^{\sigma} \underline{\delta}_{t}+\mathrm{d} \underline{\delta}_{t}^{T} \underline{K}_{\psi \psi \psi \psi}^{\sigma} \underline{\delta}_{t}+\mathrm{d} \underline{\delta}_{o}^{T} \underline{K}_{\mathrm{mm} \theta \theta}^{\sigma} \underline{\delta}_{b}+\mathrm{d} \underline{\delta}_{o}^{T} \underline{K}_{\mathrm{mm} \vartheta}^{\sigma} \underline{\delta}_{t}+ \\
& \mathrm{d} \underline{\delta}_{b}^{T} \underline{K}_{\theta \theta n \mathrm{~m}}^{\sigma} \underline{\delta}_{o}+\mathrm{d} \underline{\delta}_{t}^{T} \underline{K}_{\theta \mathrm{mm}}^{\sigma} \underline{\delta}_{o}+\mathrm{d} \underline{\delta}_{o}^{T} \underline{K}_{\mathrm{m} m \psi \psi}^{\sigma} \underline{\delta}_{t}+\mathrm{d} \underline{\delta}_{t}^{T} \underline{K}_{\psi \psi \mu m \mathrm{~m}}^{\sigma} \underline{\delta}_{o}- \\
& \mathrm{d} \underline{\delta}_{b}^{T} \underline{K}_{\theta \theta \psi \psi}^{\sigma} \underline{\delta}_{t}-\mathrm{d} \underline{\delta}_{t}^{T} \underline{K}_{\theta \psi \psi}^{\sigma} \underline{\delta}_{t}-\mathrm{d} \underline{\delta}_{t}^{T} \underline{K}_{\psi \psi \theta \theta}^{\sigma} \underline{\delta}_{b}-\mathrm{d} \underline{\delta}_{t}^{T} \underline{K}_{\psi \psi \theta \theta}^{\sigma} \underline{\delta}_{t}
\end{aligned}
$$

which may also be rewritten as follows:

$$
d U_{S}=\mathrm{d} \underline{\delta}^{T} \underline{\mathrm{K}} \underline{\delta} \quad d U_{L}=\mathrm{d} \underline{\delta}^{T} \underline{\mathrm{K}}^{\sigma} \underline{\delta}
$$

where $K$ represents the infinitesimal stiffness matrix

$$
\underline{K}=\left[\begin{array}{c|c|c}
\underline{K}_{o o o o} & -\underline{K}_{o o b b} & \underline{K}_{o o t t}-\underline{K}_{o o b t} \\
\hline-\underline{K}_{b b o o} & \underline{K}_{b b b b} & \underline{K}_{b b b t}-\underline{K}_{b b t t} \\
\hline \underline{K}_{t t o o}-\underline{K}_{b t o o} & \underline{K}_{b t b b}-\underline{K}_{t b b} & \underline{K}_{r m}+\underline{K}_{b t b t}+\underline{K}_{t t t t}-\underline{K}_{b t t}-\underline{K}_{t b t}
\end{array}\right]
$$

where $\underline{K}_{\mathrm{ijk \textrm {L }}}=\iint_{\text {element }} \underline{\mathrm{B}}_{i \mathrm{j}}^{T} \underline{\mathrm{D}}_{i \mathrm{k}} \underline{\mathrm{B}}_{k \mathrm{~L}} \mathrm{dx} \mathrm{dy}$

and $\underline{D}$ are the material stiffness matrices and $\underline{\mathrm{K}}^{\sigma}$ represents the stress stiffness matrix, which is defined as follows:

$\underline{K}^{\sigma}=\left[\begin{array}{c|c|c}\underline{K}_{m m m u m}^{\sigma} & \underline{K}_{m m \theta \theta}^{\sigma} & \underline{K}_{m m \psi \psi}^{\sigma}+\underline{K}_{m m \theta t}^{\sigma} \\ \hline \underline{K}_{\theta \theta n m}^{\sigma} & \underline{K}_{w w w w}^{\sigma}+\underline{K}_{\theta \theta \theta \theta}^{\sigma} & \underline{K}_{w w w t}^{\sigma}+\underline{K}_{\theta \theta \theta}^{\sigma}-\underline{K}_{\theta \theta \psi \psi}^{\sigma} \\ \hline \underline{K}_{\psi \psi m m}^{\sigma}+\underline{K}_{\theta m m}^{\sigma} & \underline{K}_{w w w}^{\sigma}+\underline{K}_{\theta t \theta}^{\sigma}-\underline{K}_{\psi \psi \theta \theta}^{\sigma} & \underline{K}_{w w t}^{\sigma}+\underline{K}_{\theta t \theta}^{\sigma}+\underline{K}_{\psi \psi \psi \psi}^{\sigma}-\underline{K}_{\theta \psi \psi}^{\sigma}-\underline{K}_{\psi \psi \theta t}^{\sigma}\end{array}\right]$


where $\underline{K}_{\mathrm{ijkL}}^{\sigma}=\iint_{\text {element }} \underline{\mathrm{G}}_{i \mathrm{j}}^{T} \underline{\mathrm{S}}_{i \mathrm{k}} \underline{\mathrm{G}}_{k \mathrm{~L}} \mathrm{dx}$ dy and $\underline{S}$ matrices contain the values of the stresses which need to be integrated over the thickness.

\section{Finite Force Vector}

The coupling effect of small and large effect on the strain energy density $d \bar{U}_{s L}$ can be represented as;

$$
d \bar{U}_{\mathrm{SL}}=\left(\mathrm{d} \underline{\varepsilon}_{o}-\mathrm{z} \mathrm{d} \underline{\varepsilon}_{b}+\mathrm{g}(\mathrm{z}) \mathrm{d} \underline{\varepsilon}_{t}\right)^{T} \underline{\sigma}_{L}
$$

By integration over the thickness, the strain energy per unit area is obtained as;

$$
d \mathrm{U}_{S L}^{\prime}=\mathrm{d} \underline{\varepsilon}_{\mathrm{o}}^{\mathrm{T}} \underline{\sigma}_{\mathrm{o}}-\mathrm{d} \underline{\varepsilon}_{\mathrm{b}}^{\mathrm{T}} \underline{\sigma}_{\mathrm{b}}+\mathrm{d} \underline{\varepsilon}_{t}^{T} \underline{\sigma}_{t}
$$

where $\left[\underline{\sigma}_{o}, \underline{\sigma}_{b}, \underline{\sigma}_{t}\right]=\sum_{\mathrm{m}=1}^{N} \int_{z_{L}(m)}^{z_{u}(m)}[1, \mathrm{z}, \mathrm{g}(\mathrm{z})] \underline{\sigma}_{\mathrm{L}} \mathrm{dz}$

This can be defined as shown;

$$
\begin{aligned}
& \underline{\sigma}_{o}=\underline{\mathrm{D}}_{o o}\left(\underline{\varepsilon}_{m}+\underline{\varepsilon}_{w}\right)+\underline{\mathrm{D}}_{b b} \underline{\varepsilon}_{\theta}-\underline{\mathrm{D}}_{o b} \underline{\varepsilon}_{m \theta}+\underline{\mathrm{D}}_{t t} \underline{\varepsilon}_{\psi}+\underline{\mathrm{D}}_{o t} \underline{\varepsilon}_{m \psi}+\underline{\mathrm{D}}_{b t} \underline{\varepsilon}_{\theta \psi} \\
& \underline{\sigma}_{b}=-\underline{\mathrm{D}}_{o b}\left(\underline{\varepsilon}_{m}+\underline{\varepsilon}_{w}\right)-\underline{\mathrm{D}}_{\theta b} \underline{\varepsilon}_{\theta}+\underline{\mathrm{D}}_{b b} \underline{\varepsilon}_{m \theta}-\underline{\mathrm{D}}_{\psi b} \underline{\varepsilon}_{\psi}-\underline{\mathrm{D}}_{b t} \underline{\varepsilon}_{m \psi}-\underline{\mathrm{D}}_{\theta t} \underline{\varepsilon}_{\theta \psi} \\
& \underline{\sigma}_{t}=\underline{\mathrm{D}}_{o t}\left(\underline{\varepsilon}_{m}+\underline{\varepsilon}_{w}\right)+\underline{\mathrm{D}}_{\theta t} \underline{\varepsilon}_{\theta}-\underline{\mathrm{D}}_{b t} \underline{\varepsilon}_{m \theta}+\underline{\mathrm{D}}_{\psi t} \underline{\varepsilon}_{\psi}+\underline{\mathrm{D}}_{t t} \underline{\varepsilon}_{m \psi}+\underline{\mathrm{D}}_{\psi b} \underline{\varepsilon}_{\theta \psi}
\end{aligned}
$$

where

$$
\begin{aligned}
& {\left[\underline{D}_{o o}, \underline{D}_{b b}, \underline{D}_{t t}, \underline{D}_{o b}, \underline{D}_{o t}, \underline{D}_{b t}\right]=\sum_{\mathrm{m}=1}^{N} \int_{Z_{L}(m)}^{z_{U}(m)}\left[1, z^{2}, g^{2}, z, g, z g\right] \underline{D}^{(\mathrm{m})} \mathrm{dz}} \\
& {\left[\underline{D}_{\theta b}, \underline{D}_{\theta t}, \underline{D}_{\theta \theta \psi}, \underline{D}_{\theta \psi}, \underline{D}_{\theta \theta}, \underline{D}_{\psi b}, \underline{D}_{\psi \theta \psi}, \underline{D}_{\psi t}, \underline{D}_{\psi \psi}\right]=\sum_{\mathrm{m}=1}^{N} \int_{Z_{L}(m)}^{z_{U}(m)}\left[z^{3}, z^{2} g, z^{3} g, z^{2} g^{2}, z^{4}, z g^{2}, z g^{3}, g^{3}, g^{4}\right] \underline{D}^{(\mathrm{m})} \mathrm{dz}}
\end{aligned}
$$

and

$$
g(z)=z+f(z)
$$

Hence,

$$
d U_{S L}^{\backslash}=d \underline{\delta}_{o}^{T} \underline{\mathrm{B}}_{o o}^{T} \underline{\sigma}_{\mathrm{o}}+d \underline{\delta}_{b}^{T} \underline{\mathrm{B}}_{b b}^{T} \underline{\sigma}_{\mathrm{b}}+d \underline{\delta}_{t}^{T} \underline{\mathrm{B}}_{t t}^{T} \underline{\sigma}_{\mathrm{t}}+d \underline{\delta}_{t}^{T} \underline{\mathrm{B}}_{b t}^{T} \underline{\sigma}_{\mathrm{b}}
$$

By integration over the midplane then:

$$
d U_{S L}=d \underline{\delta}_{o}^{T} \underline{\mathrm{F}}_{o o}^{L}+d \underline{\delta}_{b}^{T} \underline{\mathrm{F}}_{b b}^{L}+d \underline{\delta}_{t}^{T} \underline{\mathrm{F}}_{t t}^{L}+d \underline{\delta}_{t}^{T} \underline{\mathrm{F}}_{b t}^{L}
$$

which can be rewritten in matrix form : $d U_{S L}=d \underline{\delta}^{T} \underline{F}_{L}$

where $\underline{F}_{L}$ represents the element force vector due to coupling effect which is defined as follows:

where

$$
\underline{F}_{L}=\left[\begin{array}{lll}
\underline{F}_{o o}^{L} & \underline{F}_{b b}^{L} & \underline{F}_{t t}^{L}+\underline{F}_{b t}^{L}
\end{array}\right]^{T}
$$

$$
\underline{F}_{i j}^{L}=\iint_{\text {element }} \underline{\mathrm{B}}_{i j}^{T} \underline{\sigma}_{i} \mathrm{dxdy}
$$




\section{Geometric Nonlinear Static and Stability Analyses}

An equivalent nodal force $\underline{F}$ can be defined such that the work done by the actual applied forces due to a virtual displacement field is the same as that done by $\underline{F}$. The corresponding change of strain energy can be deduced from the strain energy as follows:

$$
d U=\mathrm{d} \underline{\delta}^{T}\left(\underline{\mathrm{K}}+\underline{\mathrm{K}}^{\sigma}\right) \underline{\delta}+\mathrm{d} \underline{\delta}^{T} \underline{\mathrm{F}}_{\mathrm{L}}
$$

Applying the principle of virtual work, then; $\mathrm{d} \underline{\delta}^{T}\left(\underline{\mathrm{K}}+\underline{\mathrm{K}}^{\sigma}\right) \underline{\delta}+\mathrm{d} \underline{\delta}^{T} \underline{\mathrm{F}}_{\mathrm{L}}-\mathrm{d} \underline{\delta}^{T} \underline{\mathrm{F}}=0$

Hence, $d \underline{\delta}$ contains arbitrary values, then; $\left(\underline{\mathrm{K}}+\underline{\mathrm{K}}^{\sigma}\right) \underline{\delta}+\underline{\mathrm{F}}_{\mathrm{L}}-\underline{\mathrm{F}}=0$ which represents the generalized equations of equilibrium

Let $\underline{\delta}+\Delta \underline{\delta}$ represent the exact solution of the equilibrium equation, then $\left(\underline{\mathrm{K}}+\underline{\mathrm{K}}^{\sigma}\right) \Delta \underline{\delta}=\underline{\mathrm{R}}$ where the residual nodal force vector $\underline{R}$ can be defined as

$$
\underline{R}=\underline{\mathrm{F}}-\left(\underline{\mathrm{K}}+\underline{\mathrm{K}}^{\sigma}\right) \underline{\delta}-\underline{\mathrm{F}}_{\mathrm{L}}
$$

This can be solved by means of iterative algorithm until acceptable value of error.

In general, the critical load is the load corresponding to large deflection, and $\underline{K}^{\sigma}$ is proportional to the stress level. Thus, a small deflection analysis can be carried out with a small load representing the distribution of actual load, and has equivalent nodal loading vector $\underline{F}_{0}$. Just before instability, the strains can always be considered infinitesimal, and if instability occurs at $\underline{F}=N_{c} \underline{\mathrm{F}}_{0}$, where Nc denotes the increase factor on stresses necessary to achieve neutral equilibrium (critical buckling load for unit force $\underline{F}_{0}$ ). This means that $\left(\underline{K}+N_{c} \underline{\mathrm{K}}^{\sigma}\right) \underline{\delta} \Rightarrow \underline{0}$ which leads to the following eigenproblem;

$$
\left|\underline{K}+N_{c} \underline{\mathrm{K}}^{\sigma}\right|=\underline{\mathrm{O}}
$$

\section{Numerical and Experimental Validation}

To ensure the accuracy of the proposed finite element derivation with buckling analysis, experimental and numerical case studies have been carried out.

\subsection{Experimental Validation}

Two types of fibre reinforced materials have been used in this work, carbon/epoxy (Fiberite 977-2 toughened epoxy resin) by Cytec Fiberite Ltd. $\mathrm{E}_{1}=128 \mathrm{Gpa}_{2}=11 \mathrm{Gpa} \mathrm{G}_{12}=\mathrm{G}_{23}=$ $\mathrm{G}_{31}=4.48 \mathrm{Gpa} v_{12}=0.25$ and layer thickness $=0.25 \mathrm{~mm}$ and glass/epoxy (Fibredux 914G-E5-30\%) by CIBA-GEIGY Ltd. $\mathrm{E}_{1}=35 \mathrm{Gpa} \mathrm{E}_{2}=8.22 \mathrm{Gpa}_{12}=\mathrm{G}_{23}=\mathrm{G}_{31}=4.1 \mathrm{Gpa} \mathrm{v}_{12}=$ 0.26 and layer thickness $=0.18 \mathrm{~mm}$. A compression testing, which was manufactured by Denison Mayes Group, has been used in the buckling test. The machine can also be used with $100 \mathrm{kN}$ as a maximum allowable load. The buckling test is applied on the two types of composites with three different dimensions. The buckling analysis is carried out by increasing the applied compression load to the buckling mode is observed.

Rectangular carbon/epoxy and glass/epoxy plates of length $180 \mathrm{~mm}$ and width $40 \mathrm{~mm}$ have been employed in the experimental tests and for the finite element package with coarse mesh (72 4-node elements). The plates have 12 layers of stacking sequence $\left[0 \% 90^{\circ} / 45^{\circ} / 0^{\circ} /-\right.$ 
$\left.45^{\circ} / 90^{\circ}\right]_{\mathrm{s}}$. Table 2 shows the critical buckling loads obtained experimentally compared with the theoretical results and with the results by ANSYS (SHELL99). It is clear from the results that the theoretical results have a good agreement with the experimental results which were slightly influenced by the effect of supports.

Table 2. Critical buckling loads

\begin{tabular}{c|c|c}
\hline \hline Material & Carbon/epoxy & Glass/epoxy \\
\hline Finite Element Package & $7964.24 \mathrm{~N}$ & $545.53 \mathrm{~N}$ \\
\hline ANSYS-12 (shell 99) & $8107.48 \mathrm{~N}$ & $551.65 \mathrm{~N}$ \\
\hline Experimental & $8100.00 \mathrm{~N}$ & $540.00 \mathrm{~N}$ \\
\hline
\end{tabular}

\subsection{Numerical Validation}

The numerical validation has been carried out to illustrate the accuracy of the proposed finite element derivation with geometric nonlinearity and stability analyses. The results have been compared with published results and with ANSYS software results.

\section{Geometric Nonlinear Static Analysis of a Clamped Free Curved Shell}

An important factor, which has to be verified before the proposed finite element program can be used safely for the analysis of shell structures, is the ability of the package to deal with composite structures with different local coordinate systems. For this purpose, a curved shell made of E-glass/epoxy has been studied numerically. The shell is clamped free with 0.1 $\mathrm{N} / \mathrm{mm}$ lateral distributed line load at the free end, $100 \mathrm{~mm}$ radius and $40 \mathrm{~mm}$ width. This case was discussed before by Attia [12], where the material properties were isotropic properties expressed as orthotropic material properties. In that case the effect of material axes in different layers does not appear as the material is actually isotropic. The shell has 8 layers $\left[0^{\circ} / 90^{\circ} /+45^{\circ} /-45^{\circ}\right]_{\mathrm{s}}$ with $2 \mathrm{~mm}$ thickness and mechanical properties as follows; $\mathrm{E}_{1}=45 \mathrm{Gpa}$ $\mathrm{E}_{2}=12 \mathrm{Gpa} \mathrm{G}_{12}=5.5 \mathrm{Gpa}$ and $v_{12}=0.19$

Two different types of meshes were attempted; coarse meshes with 40 and 80, 4-node and 3 node elements, respectively, and fine meshes with 80 and 160, 4-node and 3-node elements, respectively, as shown in Figure 3. For the purpose of validation the same meshes were also used in the ANSYS commercial package with SHELL99 element.

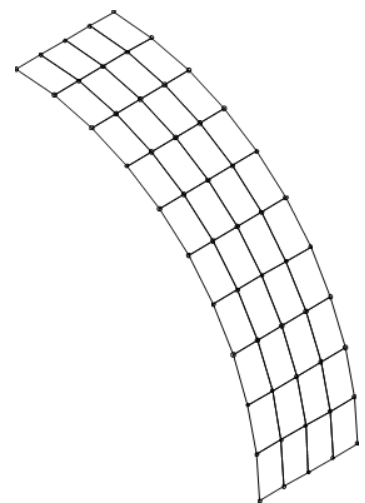

40 elements

(coarse)

4-node quadrilateral elements.

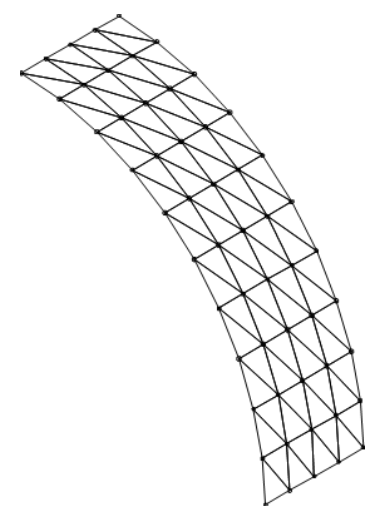

80 elements (coarse)

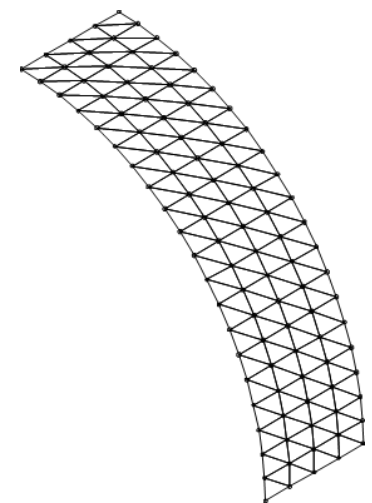

160 elements

(fine)

Fig.(2) Meshes of curved shell 


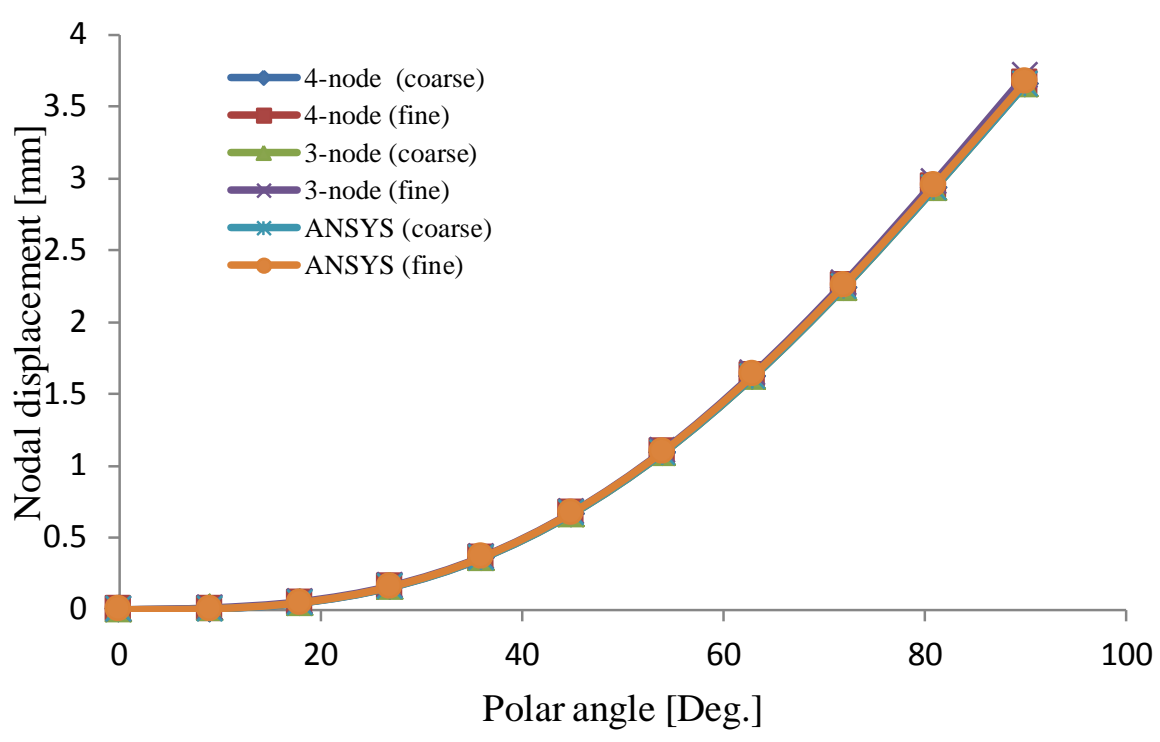

Fig. (3) Nodal deflection in z-direction versus polar angle $\theta$

Fig. (3) shows the variation of the nodal displacement shell in the load direction of the curved shell with the polar coordinates $\theta$. The results illustrate the comparison between the proposed finite element program results and the corresponding ANSYS results with coarse and fine meshes. The first observation is that the two types of meshes provide very close results to each other. It can also be seen in the figures that the package results have a good agreement with the ANSYS commercial package results. Hence, from the last two types of analyses, it is convenient to use only the 4-node elements with coarse mesh.

\section{Stability Analysis due to in-plane load}

Numerical validation of the stability analysis was carried out with two different types of stacking sequence $\left[30^{\circ}\right]$ and $\left[30^{\circ} \%-30^{\circ} / 30^{\circ}\right]$ with square and rectangular composite plates. The plates are made of E-glass/epoxy materials with the following material properties [13], $\mathrm{E}_{1}$ $=60.7 \mathrm{GPa}, \mathrm{E}_{2}=24.8 \mathrm{GPa}, \mathrm{G}_{12}=\mathrm{G}_{23}=\mathrm{G}_{31}=12.0 \mathrm{GPa} \& v_{12}=0.23$

The plates are simply supported on all four edges and subjected to in-plane uniform edge load. The buckling analysis is numerically validated with thin $(\mathrm{L} / \mathrm{t}=100)$ and thick $(\mathrm{L} / \mathrm{t}=10)$ plates with different number of layers. The buckling analysis is represented by the buckling parameter $\lambda$ as follows [13]:

$\lambda=-\frac{\mathrm{F}_{\mathrm{c}} \mathrm{L}^{2}}{\mathrm{D}_{\mathrm{o}}}$ Where $D_{o}=\frac{E_{1} \mathrm{t}^{3}}{12\left(1-v_{12} v_{21}\right)}$ and $\mathrm{Fc}$ is the critical buckling load per length.

The critical buckling load Nc is obtained by solving the eigenvalue problem. For practical purposes, the lowest mode of the eigenvalue problem is corresponding to the actual buckling load. In that case, the critical buckling load per length $\mathrm{Fc}=\mathrm{Nc} / \mathrm{b}$. The buckling parameters provided by the package are compared with the ANSYS and the published results by Narita \& Leissa [13] as shown in Table 5. The results are shown for different types of elements and different plate geometry. It can be seen from the table that the results of the package are in a good agreement with the ANSYS and the published results. 
Table 5. Buckling parameter $\lambda$ of the composite plates.

\begin{tabular}{c|c|c|c|c|c}
\hline \hline $\mathrm{L} / \mathrm{t}$ & $\mathrm{L} / \mathrm{b}$ & No. of Layers & Narita \& Leissa[13] & ANSYS (shell 99) & FEP \\
\hline \hline \multirow{4}{*}{100} & \multirow{3}{*}{1} & {$\left[30^{\circ}\right]$} & 25.17 & 25.64 & 25.19 \\
\cline { 3 - 6 } & \multirow{2}{*}{2} & {$\left[30^{\circ} /-30^{\circ} / 30^{\circ}\right]$} & 25.40 & 25.75 & 25.31 \\
\cline { 3 - 6 } & & {$\left[30^{\circ}\right]$} & 100.9 & 101.77 & 99.93 \\
\cline { 3 - 6 } & \multirow{3}{*}{10} & {$\left[30^{\circ} /-30^{\circ} / 30^{\circ}\right]$} & 101.8 & 102.51 & 100.73 \\
\cline { 3 - 6 } & \multirow{2}{*}{2} & {$\left[30^{\circ}\right]$} & --- & 22.84 & 22.01 \\
\cline { 3 - 6 } & \multirow{2}{*}{2} & {$\left[30^{\circ} /-30^{\circ} / 30^{\circ}\right]$} & --- & 22.96 & 22.13 \\
\cline { 3 - 6 } & & {$\left[30^{\circ}\right]$} & --- & 74.49 & 70.89 \\
\hline \hline
\end{tabular}

\section{Parametric Investigation}

The stiffened plates are used in several applications, such as aircrafts and ships. This section provides a parametric study for composite laminated cantilever stiffened and unstiffened plates and demonstrates the effect of some parameters on the stability analysis. The stability analysis is shown in terms of the increase factor on stresses necessary to achieve neutral equilibrium Nc (critical buckling load) under in-plane compression load. This analysis was carried out on stiffened and unstiffened plates with different number of layers to show the ability of the proposed element to deal with this type of structures and to show the effect of stiffener on the structure stability.

The stiffened cantilever plate [10] consists of four stiffeners with the same boundary conditions of the unstiffened one. Fiber orientation angles are measured with respect to the local $\mathrm{x}$-axis which is parallel to the stiffener direction. The layers are arranged in symmetry stacking sequence, such as for 4-layers plate $[\theta /-\theta /-\theta / \theta]$ and asymmetry stacking sequence, such as for 4-layers plate $[\theta /-\theta / \theta /-\theta]$. The fibre orientation angle $\theta$ has been varied from $0^{\circ}$ to $90^{\circ}$ with $15^{\circ}$ increments with two different aspect ratios $\mathrm{L} / \mathrm{b}$ (square plate $\mathrm{L} / \mathrm{b}=1$ and rectangular plate $\mathrm{L} / \mathrm{b}=2$ ) and with different number of layers.

Tables 6-7 show the critical buckling load of stiffened and unstiffened cantilever plate for carbon/epoxy and glass/epoxy, respectively. The tables illustrate the variation of the critical buckling load with the fibre orientation angles for two different aspect ratios $(\mathrm{L} / \mathrm{b}=1 \& 2)$ and with three different number of layers (4-layers, 8-layers and 12-layers).

Some observations can be summarized as, the critical buckling loads of the carbon plates are higher than the glass plates in stiffened and unstiffened cases with different number of layers. The critical buckling load of the unstiffened plate is found lower than the stiffened plates at different fiber angle. The critical buckling load is decreased by increasing the aspect ration. At the same fiber angle, the critical buckling load increases by increasing the number of layers in stiffened and unstiffened plates. The maximum values of the critical buckling load for the carbon or glass unstiffened plates are found at fiber angle $0^{\circ}$ at any number of layers with the two aspect ratios and decrease gradually as the fiber angle $\theta$ increases. The maximum critical buckling loads for the carbon or glass stiffened plates are found at different fiber angle based on the number of layers. The maximum critical buckling loads of the carbon and glass stiffened plates increase by increasing the number of layers while the fiber angle decrease or constant such as; The maximum critical buckling load of 4, 8 and 12 layers square carbon plates occurs at $45^{\circ}, 30^{\circ}$ and $15^{\circ}$ respectively. The maximum critical buckling load of 4,8 and 12 layers rectangle carbon plates occurs at $30^{\circ}, 15^{\circ}$ and $0^{\circ}$ respectively. The maximum critical buckling load of 4,8 and 12 layers square glass plates occurs at $45^{\circ}, 45^{\circ}$ and $30^{\circ}$ respectively. The maximum critical buckling load of 4,8 and 12 layers rectangle glass plates occurs at $45^{\circ}$, $15^{\circ}$ and $0^{\circ}$ respectively. 
Table 6. Critical buckling load of stiffened and unstiffened Carbon/Epoxy cantilever plate

\begin{tabular}{c|c|c|c|c|c|c|c}
\hline \hline \multirow{2}{*}{$\mathrm{L} / \mathrm{b}$} & \multirow{2}{*}{$\begin{array}{c}\text { Fiber } \\
\text { angle }\end{array}$} & \multicolumn{3}{|c|}{ Unstiffened } & \multicolumn{3}{c}{ stiffened } \\
\cline { 2 - 8 } & 0 & 165.08 & 1319.5 & 4447.0 & 5671.5 & 43446 & $1.36 \mathrm{E}+05$ \\
\hline \multirow{4}{*}{1} & 15 & 120.55 & 1102.3 & 3792.6 & 6601.9 & 63943 & $1.97 \mathrm{E}+05$ \\
\cline { 2 - 8 } & 30 & 69.448 & 673.20 & 2329.2 & 10070 & 91675 & $1.55 \mathrm{E}+05$ \\
\cline { 2 - 8 } & 45 & 33.654 & 312.62 & 1074.6 & 11539 & 57910 & 93685 \\
\cline { 2 - 8 } & 60 & 17.935 & 150.43 & 510.68 & 9791.4 & 36418 & 59137 \\
\cline { 2 - 8 } & 75 & 14.282 & 114.47 & 386.36 & 6533.4 & 24112 & 39176 \\
\cline { 2 - 8 } & 90 & 14.161 & 113.27 & 382.24 & 5034.2 & 19581 & 31826 \\
\hline \multirow{6}{*}{2} & 0 & 41.230 & 329.77 & 1112.6 & 5471.6 & 39846 & 70781 \\
\cline { 2 - 8 } & 15 & 28.291 & 268.62 & 930.54 & 6564.2 & 41905 & 66687 \\
\cline { 2 - 8 } & 30 & 14.903 & 149.69 & 520.68 & 9813.5 & 27238 & 44902 \\
\cline { 2 - 8 } & 45 & 6.9015 & 63.364 & 217.43 & 7138 & 16334 & 28808 \\
\cline { 2 - 8 } & 60 & 4.0511 & 33.561 & 113.76 & 4536.8 & 10667 & 19687 \\
\cline { 2 - 8 } & 75 & 3.5202 & 28.201 & 95.189 & 3045.2 & 7191.4 & 13412 \\
\hline
\end{tabular}

Table 7. Critical buckling load of stiffened and unstiffened Glass/Epoxy cantilever plate

\begin{tabular}{c|c|l|l|l|l|l|l}
\hline \multirow{2}{*}{ L/b } & \multirow{2}{*}{$\begin{array}{c}\text { Fiber } \\
\text { angle }\end{array}$} & \multicolumn{2}{|c|}{ Unstiffened } & \multicolumn{1}{c}{ stiffened } \\
\cline { 3 - 8 } & 0 & 9.8208 & 78.558 & 265.09 & 812.17 & 6223.2 & 20685 \\
\hline \hline \multirow{6}{*}{1} & 15 & 8.3408 & 69.699 & 236.99 & 812.74 & 7086.5 & 23966 \\
\cline { 2 - 8 } & 30 & 5.784 & 50.218 & 171.74 & 968.29 & 9113.2 & 29220 \\
\cline { 2 - 8 } & 45 & 3.7779 & 32.106 & 109.41 & 1055 & 10022 & 22741 \\
\cline { 2 - 8 } & 60 & 2.7203 & 22.17 & 75.051 & 979.88 & 8951.3 & 17193 \\
\cline { 2 - 8 } & 75 & 2.3597 & 18.907 & 63.825 & 821.4 & 6977.5 & 14157 \\
\cline { 2 - 8 } & 90 & 2.2983 & 18.386 & 62.049 & 754.65 & 5970.1 & 13077 \\
\hline \multirow{4}{*}{2} & 0 & 2.4467 & 19.573 & 66.056 & 782.22 & 6186.1 & 12411 \\
\cline { 2 - 8 } & 15 & 2.0318 & 17.184 & 58.559 & 810.23 & 6798.5 & 11521 \\
\cline { 2 - 8 } & 30 & 1.3634 & 12.035 & 41.271 & 962.27 & 5677.6 & 8956.5 \\
\hline & 45 & 0.88571 & 7.5486 & 25.735 & 1046.4 & 4028.8 & 6407.4 \\
\cline { 2 - 8 } & 60 & 0.65632 & 5.3395 & 18.07 & 974.9 & 3015.4 & 4834.7 \\
\hline & 75 & 0.58432 & 4.6805 & 15.8 & 818.78 & 2487 & 3988.1 \\
\cline { 2 - 8 } & 90 & 0.57272 & 4.5817 & 15.463 & 753.95 & 2315.9 & 3706 \\
\hline
\end{tabular}

Table 8 illustrates the critical buckling loads of 6-layers carbon and glass stiffened and the unstiffened cantilever plates with two different stacking sequences. It is clear from the results that the stacking sequence has no effect on the stability analysis for the stiffened and the unstiffened plates at fiber angles $0^{\circ}$ and $90^{\circ}$ but a small, not effective, difference in the rest of the fiber angles, $15^{\circ}, 30^{\circ}, 45^{\circ}, 60^{\circ}, 75^{\circ}$ has been observed. Observations similar to those found for the previous case can be noticed. These conclusions agree with the observations of the effect of fiber angle and number of layers investigation on the stability analysis.

Figures 4-7 show the mesh and buckling mode shapes for the unstiffened and stiffened plates with 12-layer asymmetric laminates at fiber angle $\theta=45^{\circ}$ for glass/epoxy material. 
Table 8. Critical buckling load of 6- layer stiffened and unstiffened plate with the symmetric and asymmetric stacking sequence

\begin{tabular}{|c|c|c|c|c|c|c|}
\hline \multirow{2}{*}{$\mathrm{L} / \mathrm{b}$} & & \multirow{2}{*}{$\begin{array}{l}\text { fiber } \\
\text { angle }\end{array}$} & \multicolumn{2}{|c|}{ Symmetric } & \multicolumn{2}{|c|}{ Asymmetric } \\
\hline & & & Unstiff. & Stiff. & Unstiff. & Stiff. \\
\hline \multirow{7}{*}{1} & \multirow{14}{*}{ 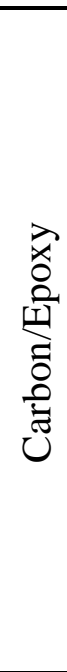 } & 0 & 556.95 & 18794 & 556.95 & 18794 \\
\hline & & 15 & 453.52 & 26329 & 445.67 & 29193 \\
\hline & & 30 & 274.57 & 43624 & 267.95 & 47425 \\
\hline & & 45 & 128.4 & 40930 & 127.61 & 40100 \\
\hline & & 60 & 62.898 & 26229 & 63.164 & 26112 \\
\hline & & 75 & 48.276 & 17400 & 48.302 & 17496 \\
\hline & & 90 & 47.79 & 13681 & 47.79 & 13681 \\
\hline \multirow{7}{*}{2} & & 0 & 139.14 & 18167 & 139.14 & 18167 \\
\hline & & 15 & 109.69 & 25515 & 108.48 & 27072 \\
\hline & & 30 & 60.663 & 19371 & 59.96 & 19092 \\
\hline & & 45 & 26.092 & 11455 & 26.023 & 11200 \\
\hline & & 60 & 14.066 & 7341.6 & 14.121 & 7344.5 \\
\hline & & 75 & 11.894 & 4921.3 & 11.902 & 4981.6 \\
\hline & & 90 & 11.938 & 4047.8 & 11.938 & 4047.8 \\
\hline \multirow{7}{*}{1} & \multirow{14}{*}{ 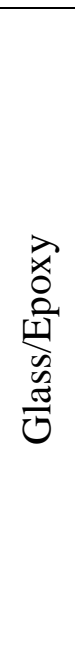 } & 0 & 33.144 & 2722.3 & 33.144 & 2722.3 \\
\hline & & 15 & 29.14 & 2938.8 & 28.965 & 3109.2 \\
\hline & & 30 & 20.844 & 3724.3 & 20.646 & 4020 \\
\hline & & 45 & 13.383 & 4094.4 & 13.314 & 4426.6 \\
\hline & & 60 & 9.3176 & 3704.3 & 9.306 & 3933.7 \\
\hline & & 75 & 7.9739 & 2924.8 & 7.9723 & 3021.8 \\
\hline & & 90 & 7.7567 & 2536 & 7.7567 & 2536 \\
\hline \multirow{7}{*}{2} & & 0 & 8.2575 & 2628.4 & 8.2575 & 2628.4 \\
\hline & & 15 & 7.1662 & 2922.5 & 7.1505 & 3091.3 \\
\hline & & 30 & 4.9788 & 3649 & 4.9678 & 3750.6 \\
\hline & & 45 & 3.1452 & 2922.2 & 3.1402 & 2859 \\
\hline & & 60 & 2.245 & 2195.8 & 2.2438 & 2189.7 \\
\hline & & 75 & 1.9741 & 1812 & 1.9738 & 1812.2 \\
\hline & & 90 & 1.9329 & 1682.1 & 1.9329 & 1682.1 \\
\hline
\end{tabular}

\section{Conclusions}

The present work contributes to the development of geometric non-linearity analysis and stability analysis of composite laminated structures using high order finite element. The proposed technique and finite element derivation have been validated by comparing the obtained results with published results and with results obtained by ANSYS commercial package for the same case studies. Good comparison with the finite element results ANSYS were observed from previous test cases, confirming the accuracy and reliability of the new derivations and the programming package.

\section{References}

[1] O. C. Zienkiewicz (1978). The Finite Element Method, Mc Graw-Hill, New York.

[2] B. Chattopadhyay, P. K. Sinha and M. Mukhopadhyay, (1995). Geometrically nonlinear analysis of composite stiffened plates using finite elements, Comp. Struc., (31): 107 118. 
[3] A. Pica, R. D. Wood \& E. Hinton, (1980). Finite element analysis of geometrically nonlinear plate behaviour using Mindlin formulation, Computer \& Structures, (11): 203-215.

[4] R.D. Mindlin, (1951). Influence of rotary inertia and shear on flexural motions of isotropic elastic plates, J. of Applied Mechanics, (18): 31-38.

[5] E. Reissner, (1945). Reflection on the theory of elastic plates, J. of Applied Mechanics, (38):1453-1464.

[6] Ferreira A. J. M. and Barbosa J. T. (2000). Buckling behaviour of composite shells, Comp. Struc., (50): 93-98.

[7] Istvan A. V. and Laszlo P. K. (2001). Buckling of rectangular orthotropic plates subjected to biaxial normal forces, Journal of Composite Materials, 35-(7): 625-635.

[8] M. Bodaghia and A.R. Saidi (2010). Levy-type solution for buckling analysis of thick functionally graded rectangular plates based on the higher-order shear deformation plate, theory Applied Mathematical Modelling, 34-(11):3659-3673

[9] Lars Fiedler, Walter Lacarbonara and Fabrizio Vestroni (2010). A generalized higherorder theory for buckling of thick multi-layered composite plates with normal and transverse shear strains, Comp. Struc., 92-(12):3011-3019

[10] Moutaz M. Hegaze (2010). Nonlinear dynamic analysis of stiffened and unstiffened laminated composite plates using a high-order element, J. of Composite Mat. , 44(3):327-346.

[11] Timoshenko, S. P. \& Gere J. M. (1961). Theory of Elastic Stability, Mc Graw-Hill Book Company, Inc.

[12] Attia O. (1996) "Finite element static, dynamic, and flutter analysis of rotating composite layered plates and shells" Ph.D. Thesis, SME, Cranfield University.

[13] Narita Y. and Leissa A. W. (1990). Buckling studies for simply supported symmetrically laminated rectangular plates, International Journal of Mechanics and science, 32-(11): 909-924.

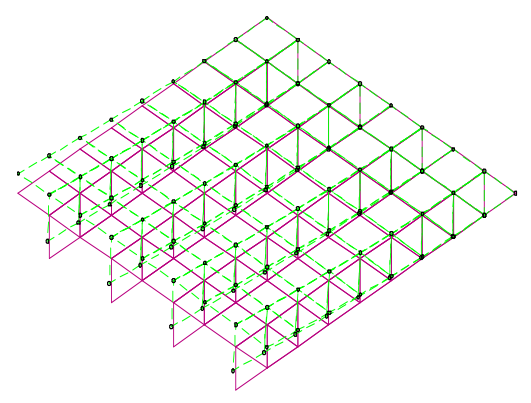

Fig.(4) Buckling mode of 12-layer stiffened square cantilever plate

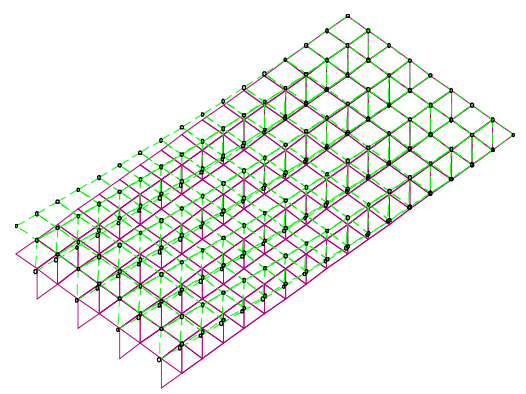

Fig. (6) Buckling mode of 12-layer stiffened rectangular cantilever plate

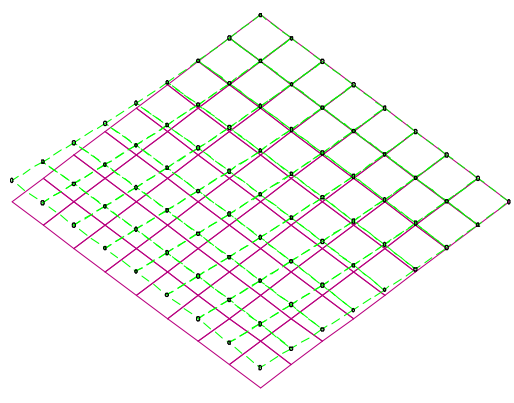

Fig.(5) Buckling mode of 12-layer unstiffened square cantilever plate

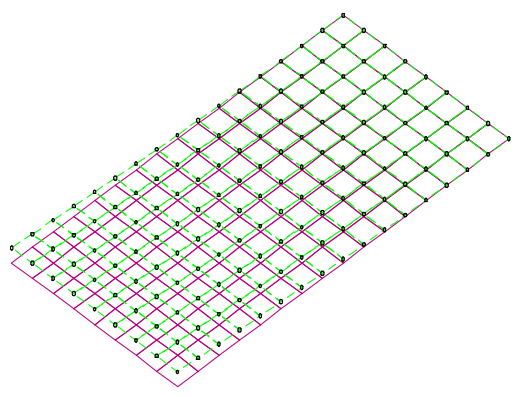

Fig. (7) Buckling mode of 12-layer unstiffened rectangular cantilever plate 